\title{
Géolinguistique
}

$17 \mid 2017$

Varia

\section{Réflexion géolinguistique sur le mot sel}

Geolinguistic Reflection on the Word Sel

\section{Yuji Kawaguchi}

\section{CpenEdition}

Journals

Édition électronique

URL : http://journals.openedition.org/geolinguistique/355

DOI : $10.4000 /$ geolinguistique.355

ISSN : 2650-8176

\section{Éditeur}

UGA Éditions/Université Grenoble Alpes

\section{Édition imprimée}

Date de publication : 1 décembre 2017

Pagination : 7-22

ISBN : 978-2-37747-025-9

ISSN : 0761-9081

\section{Référence électronique}

Yuji Kawaguchi, «Réflexion géolinguistique sur le mot sel », Géolinguistique [En ligne], 17 | 2017, mis en ligne le 01 février 2019, consulté le 30 octobre 2020. URL : http://journals.openedition.org/ geolinguistique/355; DOI : https://doi.org/10.4000/geolinguistique.355 


\title{
Réflexion géolinguistique sur le mot sel
}

\author{
Yuji Kawaguchi \\ Tokyo University of Foreign Studies
}

\section{Résumé}

Autour du mot sel, notre argument s'est focalisé sur la consonne /-1/ finale dont la chute est attestée dès la fin du XIII ${ }^{\mathrm{e}}$ siècle. Le maintien de la consonne finale était la norme chez les poètes depuis le Moyen Âge, alors que sa suppression traduisit une prononciation populaire comme l'indiquent clairement les témoignages des grammairiens. Pendant plusieurs siècles, l'absence de /-1/ a coexisté avec /-1/ prononcée. Depuis le XIX ${ }^{\mathrm{e}}$ siècle, sous la pression croissante de la capitale, la forme sel va envahir au moins la France du Nord, et la forme sé ne pourra survivre que dans les régions qui demeurent à l'abri des vagues de standardisation du français.

\section{Mots-clés}

Sel, -1 finale, ALF, ALB.

\section{Abstract}

As for the word sel, our argument focuses on the dropping of the final consonant /-l/, which was attested at the end of the thirteenth century. Pronouncing the final /-l/ was the norm among poets since the Middle Ages, while /-l/ dropping was the popular pronunciation of ordinary people, as clearly indicated by the testimony of the grammarians. For several centuries, the pronouncing and dropping of /-l/ were both observed. Since the 19th century, under the increasing pressure of standardization from the capital, sel has prevailed in the Northern France area, limiting the survival of the form se only to the regions intact from the waves of standardization.

\section{Keywords}

Salt, final -l, ALF, ALB. 


\section{Introduction}

D'après la description de Meyer-Lübke (1895 : 467-469), le mot sel issu du latin SĀL connaît une variation intéressante de genre parmi les langues romanes. Il est masculin en français, italien, portugais, rhéto-roman et frioulan, tandis qu'il est féminin en occitan, catalan, espagnol et roumain. À première vue, la différence de genre se trouve coïncider avec la scission entre français et occitan. Au sujet de ce fameux assaisonnement indispensable à la cuisine, Jules Gilliéron et Jules Mongin furent probablement les premiers à traiter de sa situation géolinguistique en France. Leur étude de la géographie linguistique du mot se fondait sur les trois points suivants.

Premièrement, partant du principe que «la coïncidence ne peut pas être naturelle» (Gilliéron \& Mongin, 1907 : 293), ils critiquent l'explication simpliste du masculin le sel en langue d'oïl et du féminin la sau en langue d'oc, (voir fig. 1). En effet, la zone rouge du genre féminin dépasse le domaine d'oïl oriental et la frontière entre français et occitan. Ils ajoutent que «l'Est nous apporte le trait de lumière. Entre l'aire la sau et l'aire le sé - le sel s'étend une aire le sau» (ibid.). Il faut cependant remarquer qu'ils emploient une seule forme sau pour deux réalités phonétiques différentes. La forme sau représente [saw $]^{1}$ ou [saw] dans le Midi, mais [so:] ou [so:] à l'est de la France. La dernière forme se propage non seulement dans le nord du domaine franco-provençal, englobant Jura, Doubs et Suisse romande, mais aussi en Haute-Sâone et dans les Vosges.

Le second argument concerne l'analyse géolinguistique. Gilliéron et Mongin analysent la distribution géographique de sé et sel dans le domaine d'oïl en se basant sur le principe des aires latérales établi par Jakob Jud et Matteo Bartoli (Dauzat, 1944 : 45). La première forme sé témoigne d'une couche plus ancienne, car elle se maintient habituellement dans les périphéries du Nord-Ouest, du Nord et du Nord-Est. Et c'est la nouvelle forme sel qui a remplacé sé dans la plupart des points du domaine d'oïl, sé n'étant que sporadiquement attestée dans l'Yonne, l'Aube et la Nièvre.

Le troisième argument résulte logiquement du deuxième. Si la forme sé est considérée plus ancienne, il est nécessaire d'expliquer l'origine de l'invasion de sel. Gilliéron et Mongin font appel à l'hypothèse d' «une restauration artificielle, due à l'action probable des formes devant voyelle (le sel est)» (1907 : 294). On peut alors se demander pourquoi la position antévocalique fut privilégiée seulement pour restituer la consonne /-1/ finale pour le mot sel, mais non pour d'autres mots tels que cul, outil, persil et soûl.

1. On retranscrit les symboles phonétiques de l'ALF en leurs correspondants approximatifs de l'API. 
Peut-on sérieusement soutenir que sé était particulièrement menacé, par exemple exposé au danger de collisions homonymiques (Thibault, 2017)?

\section{Brun-Trigaud, Le Berre et Le Dû (2005)}

En l'état actuel de notre connaissance, la carte interprétative du sel la plus récente (voir fig. 2) se retrouve dans un excellent vadémécum publié par trois dialectologues français. Le manuel a pour but de donner une orientation consistante pour la lecture des cartes de l'ALF. Il faut remarquer tout abord que la figure 2 corrige la figure 1 de Gilliéron et Mongin. Il s'agit de l'absence de la forme [la sal] ou [la sal] dans la figure 1. Le maintien de la consonne /-1/ est un trait proéminent du languedocien (Bec, 1983: 20). D'autre part, comme je l'ai déjà souligné, dans l'est de la France en particulier, la frontière entre le sel et la sau remonte nettement au nord de la frontière des langues d'oïl et d'oc. Mais ici aussi, à moins que la forme de l'est le sau [1 so/o] ne soit confondue avec la sau [la saw] du Midi, il vaudrait mieux noter le só/sò au lieu de le sau dans la figure $2^{2}$.

Le manuel indique en outre une raison extralinguistique à la propagation de la forme sel dans le nord de la France. Autrefois, la gabelle était un impôt royal sur le sel. Le système de la gabelle exista jusqu'à la Révolution, réapparut sous Napoléon Ir et fut définitivement aboli en 1945. En vue de percevoir des gabelles d'une manière plus efficace, une ordonnance de mai 1680 ratifia la division du royaume en six pays soumis à des règles différentes de perception (voir la fig. 3 qui dessine la situation au moment de la Révolution).

Les pays de petite gabelle recouvrent les domaines actuels du Languedoc, du Massif Central et du Dauphiné. Les provinces rédimées de gabelles embrassent le Poitou, le Limousin, l'Auvergne et la Gascogne. La Bretagne était le pays de franc-salé et la Basse Normandie était celui de quart-bouillon. Alsace et Franche-Comté constituaient des pays de salines. Enfin, les pays de grande gabelle comprennent, du nord au sud, Picardie, Haute-Normandie, Champagne, Île-de-France, Orléanais, Maine, Anjou, Touraine, Berry et Bourgogne. Ils englobaient un domaine central très étendu de la France. Brun-Trigaud, Le Berre et Le Dû remarquent que "cette propagation de "le sel" s'éclaire nettement lorsqu' on effectue un rapprochement avec l'aire de la grande gabelle [...]. Les percées de

2. Sur la carte 1213 de l'ALF, on observe assez fréquemment une autre variante [sā] ou [sa/a] près de [saw] ou [so/o]. 
la forme sel à l'embouchure de la Loire et vers Saint-Malo trouvent sans doute leur explication dans le commerce du sel» (2005: 139). Ces pays de grande gabelle correspondent grosso modo à la zone où n'existe plus aucun parler nettement différencié du français (Pottier, 1969 : 1156) ou à la zone centrale dépourvue de nombre des isoglosses établies par Arvid Rosenqvist (1919; Berschin, Felixberger \& Goebl, 2008 : 261).

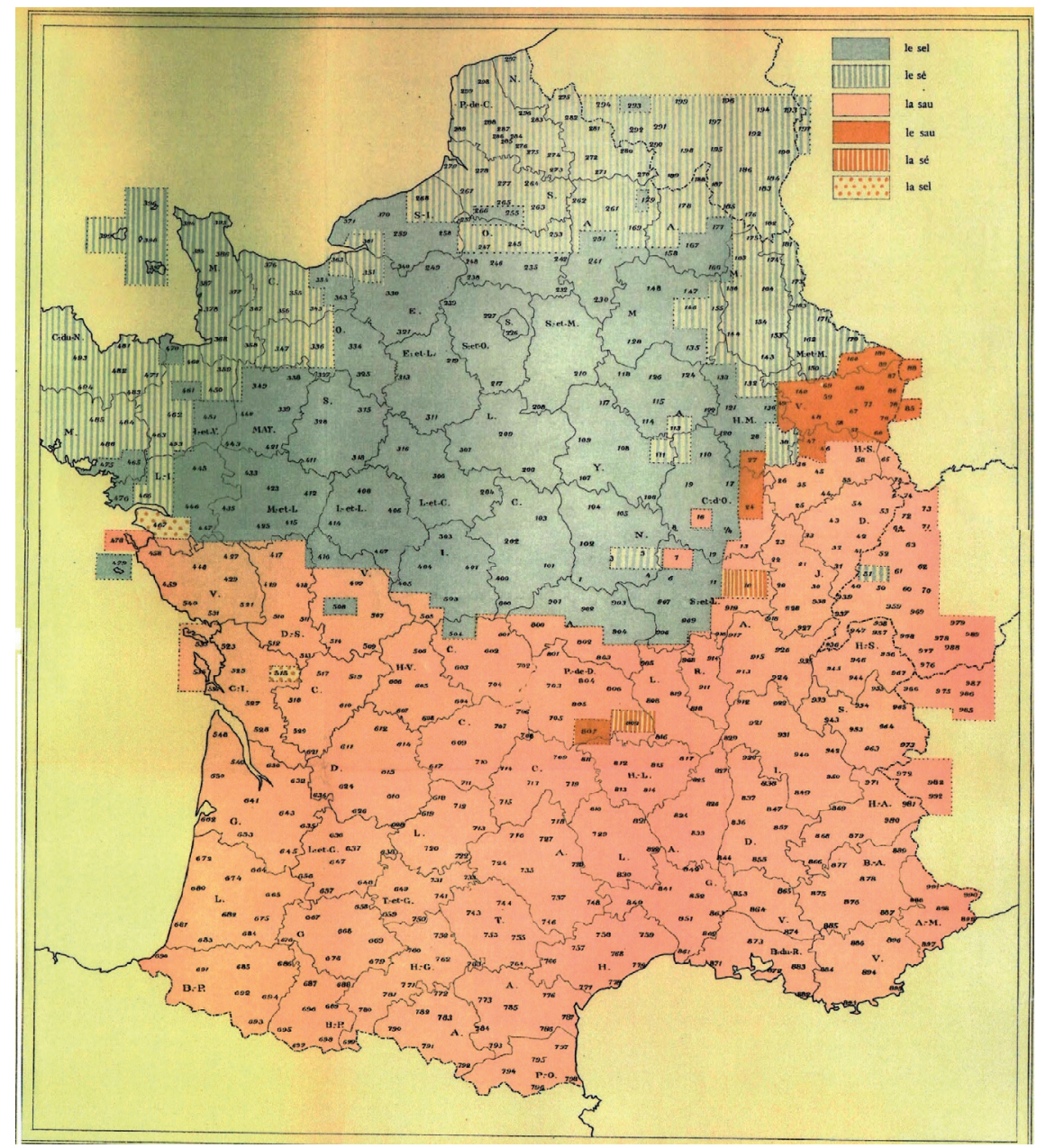

Figure 1. - ALF Piler LE SEL.

(Gilliéron \& Mongin, 1907) 


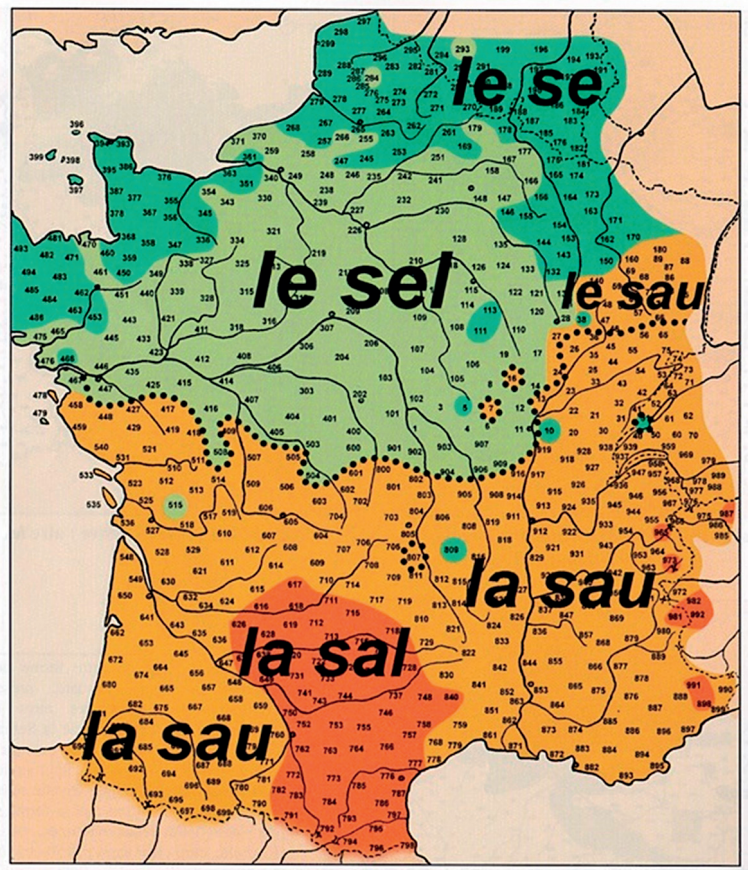

Figure 2. - ALF 1213 le sel.

(Brun-Trigaud, Le Berre \& Le Dû, 2005 : 138)

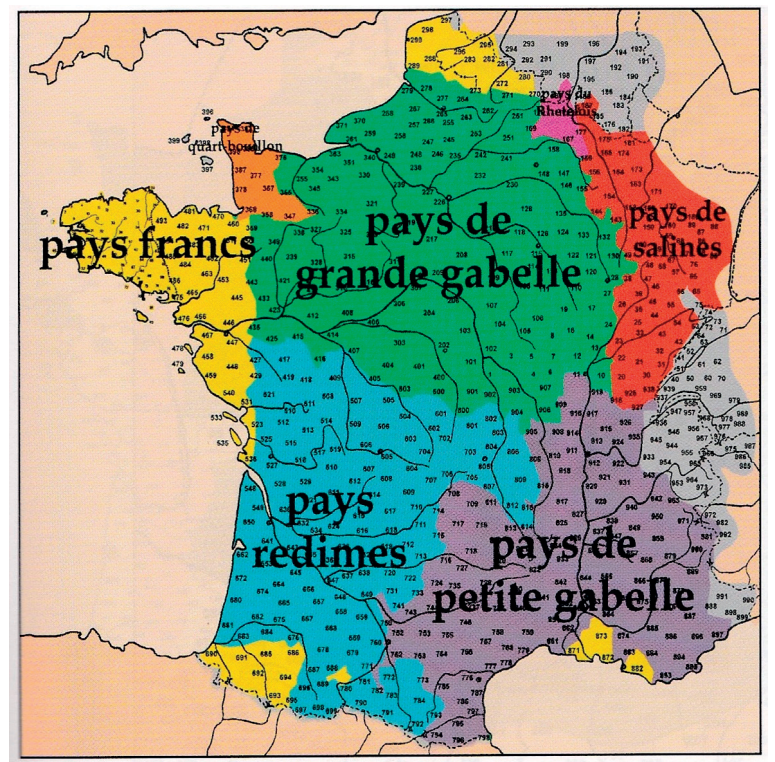

Figure 3. - Carte de pays de gabelles (1789) (Brun-Trigaud, Le Berre \& Le Dû, 2005 : 139). 


\section{Diachronie de la consonne /-I/ finale}

D'après la phonétique historique traditionnelle, la consonne /-1/ finale reste telle quelle après les voyelles latines $/ \overline{\mathrm{A}} /$, /Ě $/$ et $/ \overline{\mathrm{O}} /$ comme TĀLIS $>t e l$, MĚL $>$ miel, sōLum $>$ seul, alors qu'elle tombe après $/ \bar{U} /$ comme CốLum $>$ cul et /I// comme * GĚNTĪLEM $>$ gentil (Regula, $1955: 129)$. Toutefois, l'évolution des consonnes finales n'est pas si simple, loin de là. On peut relever les phénomènes suivants en français moderne : hésitation dans la prononciation entre [ut] et $[\mathrm{u}]$ pour août, dans l'orthographe entre clé et clef, variation dans la prononciation entre aimer et finir, existence du doublet $\mathrm{cou} / \mathrm{col}$, histoire complexe de sceau et des formes disparues seel et scel. Dans la discussion qui suit, nous nous bornerons cependant à la consonne /-1/ finale.

À la fin du XIII ${ }^{\mathrm{e}}$ siècle, Rutebeuf utilisait les rimes suivantes qui attestent la chute de /-1/ pour le mot ostel :

Mes ostes veut l'argent avoir / De son osté (= ostel), / Et j'en ai presque tout osté, / Et si me sont nu li costé / Contre l'yver. (Rutebeuf, Complainte Rutebeuf, v. 75-79, BN. fr. 837)

«Mon propriétaire veut toucher le loyer / de la maison, / et je l'ai presque entièrement vidée, / je suis nu / face à l'hiver:» (Zink, 1989 : 291)

Dans un autre manuscrit originaire de l'est, datant du XIV siècle, le copiste conserve la consonne /-1/ pour le même mot dans les rimes en -ei.

Mes hostes wet l'argent avoir / De mon hosteil, / Et j'en ai presque tout ostei, / Et si me sunt nu li costei / Contre l'iver. (Ouvr. cité, BN. fr. 1635)

À la première moitié du XIve siècle, sous la plume d'un poète et ménestrel originaire de Couvin, près de Namur, on trouve les rimes suivantes :

S'en vint chascuns a son osté (= ostel), / Son hiaume et son escu osté, [...]

(Watriquet de Couvin, Dits, XVII Li Mireoirs aus princes, v. 243-244)

«chacun s'en vient à sa maison, / son casque et son écu enlevés, [...]»

Le mot issu du latin HǒsP̌TĀLIS connaissait donc une variation entre osté et ostel au Moyen Âge. Rutebeuf témoigne aussi de la forme tronquée té pour le mot tel issu du latin TĀLIS.

Nus n'i prent més s'il n’y a mis : / Qui riches est s'a parenté; / Més povres hom n'a parent té (= tel), / S'il le tient plus d'une jornee, / Qu'il ne plaingne la sejornee. (Rutebeuf, Les Plaies du monde, v. 16-20, BN. fr. 837)

«Nul n'a de parents s'il n'y a mis le prix : / qui est riche a de la parentèle, / mais le pauvre n'a de parent tel / qu'il ne plaigne les frais / s'il reste chez lui plus d'un jour.» (Zink, 1989 : 69) 
Citons encore la laisse suivante dans la chanson de geste d'Aye d'Avignon composée vers 1200 :

[...] Onques n'en sorent mot li baron naturé (= naturel), / Si se virent enclos de . $V^{c}$. ferarmé. [...] (Aye d'Avignon, v. 845-846)

«[...] les hommes de bonne race ne purent émettre un mot, et se virent entourés de cinq cents ennemis armés $[\ldots]$ »

Le témoignage de la forme sé pour sel ne semble pas attesté en ancien français. FEW enregistre sau comme la forme du moyen français et une forme poitevine [sau] du XIV $\mathrm{XVV}^{\mathrm{e}}$ siècles (FEW digital, vol. 11, p. 76). L'absence de sé en ancien français semble obscure, probablement due à la différence étymologique : -ĀL pour sel, mais -ĀLIS pour ostel, tel, naturel. Quoi qu'il en soit, il est incontestable que la variation entre -el et -é s'est produite pendant la période du Moyen Âge.

En ce qui concerne l'état du $X v^{e}$ et du début du $X V I^{e}$ siècle, sur la base de nombreux exemples de poèmes de Charles d'Orléans, Mystère du siège d'Orléans et les Soties, Pierre Fouché conclut que la chute de consonnes finales s'intensifiait avec le temps. Tout en admettant que la grande majorité des rimes indiquent que les consonnes finales étaient prononcées par les poètes à la fin du $\mathrm{Xv}^{\mathrm{e}}$ siècle, il remarque que «d'autres, plus rares, sans consonne finale écrite ou avec consonne finale écrite au rebours, supposent une adoption du phonétisme vulgaire commandée par les besoins de la rime, c'est-à-dire la chute» (Fouché, $1961:$ 664).

À partir de l'aube du $\mathrm{XVI}^{\mathrm{e}}$ siècle, on dispose de témoignages métalinguistiques sur la consonne /-1/. Dans les lignes qui suivent, pour mieux faire ressortir le parallèle avec sel, la consonne /-1/ sera analysée généralement dans des mots monosyllabiques, et deux contextes phonétiques seront soigneusement distingués : /-1/ après les voyelles autres que /i/ et /-1/ après la voyelle /i/.

\section{Témoignages chez les grammairiens}

\section{1. /-I/ après les voyelles autres que /i/}

La consonne $/-1 /$ reste intacte devant les voyelles $/ \mathrm{a} /, / \varepsilon /, / \mathrm{\rho} /{ }^{3}$ et $/ œ /$ comme $\mathrm{mal}$, sel, bol, seul, bien que dans un cas sa vocalisation ait donné naissance au doublet col/cou. D'après Laurent Chifflet (1680 : 238), la consonne /-1/

3. «Autrement elle (=/-1/) se prononce après les voyelles $a, e, o, \& u$ :» (Oudin, 1640 : 23) 
se prononce toujours comme dans tel et fel. Pierre de La Touche (1760: 25) préconisait que la consonne /-1/ sonne à la fin du mot fel. La forme sel a été utilisée comme exemple par des grammairiens (Buffier, $1714: 396$; Boyer, 1794 : 14; Malvin-Cazal, 1846 : 397). Mais à côté de ces commentaires recommandant d'articuler /-1/, plusieurs témoignages de l'absence de /-1/ sont attestés dès le $\mathrm{XVI}^{\mathrm{e}}$ siècle. Lorsque John Palsgrave explique comment prononcer les vers d'Alain Chartier, il tolère la lecture morté $(=$ mortel $)$.

Après maint dueil, et maint mortel péril,

Apremayndveíl, emaynmortéperil, (Palsgrave, 1852 [1530] : 60)

Il autorise de même la lecture leké (= lequel) dans L'Illustrations de Gaule de Jean Lemaire de Belges (1473-1524) :

lequel je cognois par la démonstration de ta phisonomie,

lekéjeconóaparlademovnstratióvndetafizonomió, (ouvr. cité : 62)

D'autres exemples révèlent explicitement la chute de /1/ antéconsonantique dans quel au XVI ${ }^{\mathrm{e}}$ siècle. Faisant référence au registre familier, Claude Buffier écrit que «L finale. Se prononce : sel, royal, fil, b mol; exceptez baril, chenil, nombril,persil, \& les pronoms il \& quel suivis d'une consonne dans le discours familier : persil, il dit, quel monstre, \&c. prononcez persi, i dit, qué monstre :» (1732 : 202-203 et 914-915)4. Eléazar de Mauvillon est aussi témoin de la même variation.

Le P. Buffier prétend que dans le discours familier, on ne fait point sentir $l^{\prime} l$ dans quel, quand il suit une consonne; il est vrai qu'il y a des personnes, qui font entendre ce son dans quelques mots \& prononcent qué conte, qué chien de portrait; mais c'est qu'en parlant vite, ces personnes coulent si légérement sur l'l qu'elles semblent ne pas la prononcer. À cela près il n'y a que le petit peuple qui prononce qué conte, qué drille! (Mauvillon, $1754: 67)$

On a affaire ici à la variation sociolinguistique de la consonne /-1/ devant un mot débutant par une consonne. D'autre part, Charles Bovelles insiste sur la variation géolinguistique du mot Noël: «Noel, alors comme nos Belges disons Nouel, [...] les Parisiens disent Noel, les Amiénois Noue : nos gens de la Somme plus proches du latin font allusion à Nauel ${ }^{5}$.» $(1533: 70)$ Claude de Saint-Liens (1566 : 79) explique aussi la variante Noé pour Noel.

4. Étrangement Buffier n'avait pas introduit cette variation du discours familier dans sa Grammaire françoise (Buffier, 1709 : 389-390 et 914-917).

5. «Noel, vel sicut nos Belgae loquimur, Nauel, per V vocalem, id est celebritas Dominicae natiuitatis. tractum est à voce natale, suppressa, vt fieri persępe solet in medio, 
Nathanaël Duez (1669 : 53) attire l'attention sur la chute du /-1/ de Noël et Michel. La nature de cette variation ne paraît pas très simple, bien qu'un contraste apparaisse à première vue soit entre lettrés et petit peuple, soit entre style soutenu et style familier, soit entre Paris et régions. En effet, dans son cahier daté du 28 juin 1605 (Ernst, 1985 : 180), Jean Héroards, docteur du dauphin, futur Louis XIII, remarque même dans la parlure du dauphin la collision homophonique de tout mal et Thomas. Quoi qu'il en soit, la forme -el avait une forte chance de perdre sa consonne finale soit devant pause soit devant consonne à partir du XVI ${ }^{\mathrm{e}}$ siècle au moins et jusqu'au $\mathrm{XVIII}^{\mathrm{e}}$ siècle. Et on ne peut plus trouver la trace de /-1/ tombée dans les deux dictionnaires de prononciation de Féline (1851) et de Michaelis et Passy (1914 [1897]).

\section{2. /-I/ après la voyelle /i/}

La trace de cette variation sociolinguistique apparaît le plus clairement après la voyelle /i/. Selon Claude Irson (1662 : 27), la consonne /1/ forte se prononce comme dans fil, subtil. L'observation de Thurot sur le mot fil est ici pertinente : «On ne dit jamais autrement que fil par une $l, \mathrm{H}$. Estienne. L' $l$ est muette devant une consonne ou une pause, Martin 37; "fil ne se prononce qu'en cette phrase : de fil en aiguille", Chifflet; l'l est toujours muette, Duez. [...].» (1881: 142) On recense d'autres attestations dans Thurot (1881). Odet de Lanoue tolère chenil et cheni, mais /-1/ finale est muette selon Jean Hindret. La consonne /-1/ de gril est muette chez Henri Estienne, tandis que d'après Lanoue, elle se prononce. François-Séraphin Régnier-Desmarais et Pierre de La Touche disent qu'on pourrait prononcer /-1/ dans les vers. L'Académie 1719-1740 édicte que /-1/ ne se prononce qu'en vers et devant une voyelle. L'Académie 1769 change finalement la norme et conseille de ne point prononcer /-1/ dans le discours familier. En réalité, cette consonne était mouillée quand on la prononçait après /i/.

Ainsi, la norme divergeait fortement chez les grammairiens. Le pronom il l'illustre bien, comme l'explique Henri Estienne sur une base sociolinguistique.

Quinetiam non deesse scio qui hoc in loco, Puisqu'il t'a pleu, nullum literae $l$ sonum darent in particula $i l$ : sed pronuntiarent, Puisqu'i t'a pleu : verum haec ab imperito profecta vulgo pronuntiatio, plane est explodenda : (Estienne, 1582: 80)

litera duriuscula T, vel versa ea in V vocalem. Parrhisii dicunt Noel, Ambiani Noue : nos Samarobrini propius voci Latinae alludentes Nauel.» 
«De plus, je sais qu'il ne manque pas de gens qui ne prononcent pas l' $l$ du mot $i l$ dans le cas de Puisqu'il t'a pleu : mais cette prononciation, Puisqu'i t'a pleu, en effet venue du peuple inexpérimenté et vulgaire, doit complètement être rejetée.»

L'Académie adopte également ce point de vue sociolinguistique à partir de la seconde moitié du XVIII ${ }^{\mathrm{e}}$ siècle. La fluctuation persiste jusqu'au XIX ${ }^{\mathrm{e}}$ siècle. Dans son Dictionnaire de la prononciation (1851), Adrien Féline décrit [avril], [bari], [nonbri-1] ${ }^{6}$. Les deux éditions du Dictionnaire phonétique de Michaelis et Passy attestent l'évolution de la norme de la prononciation : [avril] ou [avri] dans la première édition de 1897, mais [avril] seulement dans la deuxième édition de 1914. De même, on a [bari] pour baril dans la première édition, mais [bari] ou [baril] dans la deuxième; [nõbri] nombril reste inchangé. Au XIX ${ }^{\mathrm{e}}$ siècle à travers le progrès de la scolarisation, le prestige de l'orthographe a eu une influence sur la prononciation des consonnes finales.

Quelle conclusion peut-on tirer de tout ceci? Tout d'abord, compte tenu des rimes utilisées par Rutebeuf, que la chute de /-1/ finale est attestée à Paris dès la fin du XIII ${ }^{\mathrm{e}}$ siècle. La même consonne a pu se vocaliser pour donner la forme sau pour sel du moyen français. Le doublet col et cou provient vraisemblablement d'une telle fluctuation de /-1/ finale. Le maintien de la consonne finale représente, comme le suggère Fouché, la norme chez les poètes à la fin du $\mathrm{Xv}^{\mathrm{e}}$ siècle, la suppression de /-1/ n'étant qu'une adoption du phonétisme vulgaire. La même tendance persiste après le $\mathrm{XVI}^{\mathrm{e}}$ siècle. Grâce aux témoignages des grammairiens, on peut faire l'hypothèse que la chute de /-1/ n'est pas un cas de variation libre, mais un fait sociolinguistique. Il s'agit d'une variation corrélée aux clivages sociaux. La forme sans /-1/ était probablement emblématique de la parlure populaire. Cependant, il vaudra mieux s'en tenir ici à une variation liée aux différences de registres, une même personne pouvant alors se servir des deux variantes suivant la situation de communication. En observant les mots qui finissent par -il, on peut imaginer plus précisément une telle fluctuation, qui a persisté pendant plusieurs siècles. Il s'ensuit que, malgré l'opinion de Gilliéron et d'autres chercheurs (Straka, 1981 : 242), le maintien de la consonne /-1/ finale ne serait dû ni à la restitution savante basée sur le latin, ni à la consonne $/ 1 /$ de liaison avant un mot commençant par voyelle.

6. - 1 est la forme de liaison. 


\section{Discussion}

Environ vingt ans après la publication de l'ALF, Albert Dauzat lança un nouveau projet d'atlas linguistiques de la France par régions (voir SimoniAurembou, 2004 et Brun-Trigaud, 2016). Les travaux des dialectologues français se sont nourris de l'ensemble des Atlas linguistiques et ethnographiques des diverses régions (ALFR). Jean-Philippe Dalbera a vu dans l'ALF le fruit d'une dialectologie de la première génération, et dans l'ALFR celui de la deuxième génération (Dalbera, 2007 : 43-44). L'ALFR vise à améliorer la méthodologie de l'ALF et à accumuler plus de données dialectales à travers des réseaux d'enquête plus serrés et approfondis. Dans cet exposé, nous voudrions mettre en relief l'innovation et la persistance des dialectes entre deux atlas linguistiques, l'ALF et l'ALB (Atlas linguistique et ethnographique de la Bourgogne), séparés par quelque soixante ans.

La carte interprétative de l'ALB $n^{\circ} 1494$ du sel est donnée dans la figure 4. Les formes féminines symbolisées en noir sont attestées dans les régions plus ou moins conservatrices de la Bourgogne, c'est-à-dire dans la moitié sud-est de la Côte-d'Or et la moitié est de la Saône-et-Loire (Taverdet, $1973: 320-323$ ).

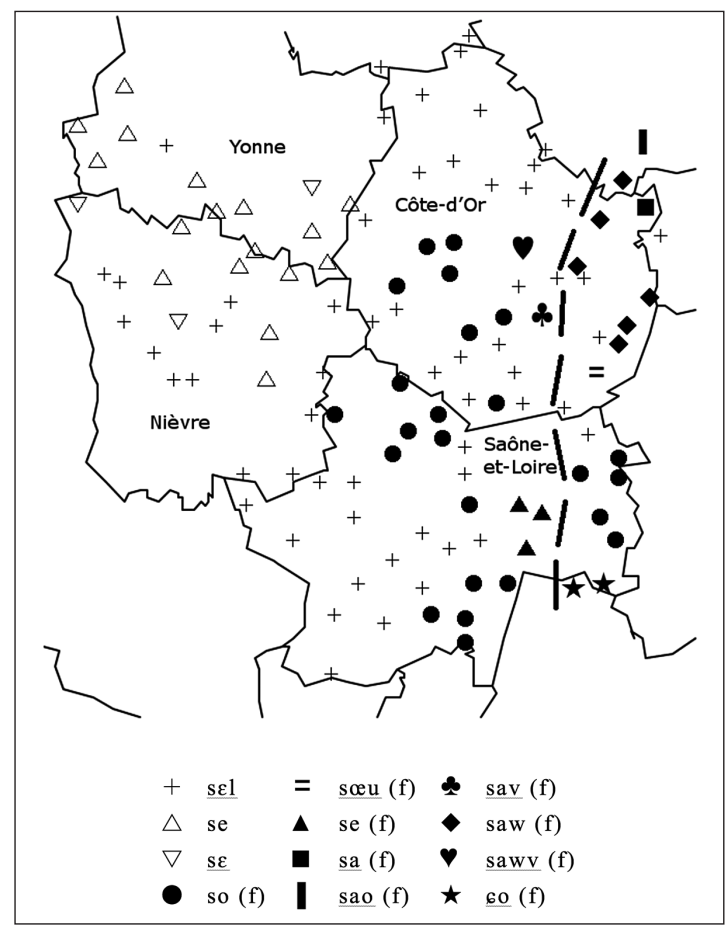

Figure 4. - ALB 1494 du sel. 
La frontière orientale de la forme standard sel dans l'ALF est marquée par une ligne brisée. Il est évident qu'une pénétration de sel a eu lieu dans l'est pendant un demi-siècle. À l'opposé de l'ALF, une large zone de [se] représentée par $\Delta$ et de [sc] par $\nabla$ s'étend en Puisaye et dans le Morvan. Cette découverte résulte de l'enquête plus fine et minutieuse de l'ALB. La zone en question dépasse largement la Bourgogne en s'étendant dans le sud de l'Yonne en Champagne. La persistance de sé dans une région non éloignée de Paris peut avoir contribué, d'une part, à la variation sociolinguistique entre $s e l$ et sé dans le langage parisien pendant plusieurs siècles et, d'autre part, à la survivance de sé, ancienne forme populaire, dans les dialectes modernes. Une telle perspective n'est pas du tout nouvelle; elle remonte aux principes de la géographie linguistique du siècle dernier. Gilliéron se demandait dès sa première étude géolinguistique « $\mathrm{Si}$ les événements ne se sont pas enchaînés ainsi dans l'histoire, pourquoi s'enchaînent-ils géographiquement?» (Gilliéron \& Mongin, 1906 : 85). La continuité tant chronologique que géographique est patente dans notre cas aussi. La forme standard sel dans l'ALF a progressé petit à petit vers l'est dans l'ALB. Nous ferons l'hypothèse suivante sur la progression de $s e l$ : l'ancienne variation sociolinguistique entre sel et sé disparaîtra progressivement au profit de la norme sel, en même temps que sé continuera à survivre dans des zones plus à l'abri de l'influence de la langue standard.

Le présent exposé a mis en évidence l'importance des témoignages métalinguistiques pour l'analyse géolinguistique. À l'époque de Gilliéron, où on était encore à l'aube de la géographie linguistique, l'objectif principal des recherches était de se construire une base scientifique. Pour ce faire, Gilliéron était plus ou moins obligé de minimiser la valeur des apports écrits. "Mais quel que soit l'intérêt des vérifications après coup que les recherches doivent rencontrer dans les textes, elles ne peuvent qu'illustrer, non confirmer, le résultat auquel aboutit la méthode géographique.» (Gilliéron \& Mongin, 1907 : 296) En la matière, l'auteur de ces lignes serait plutôt d'accord avec Dauzat : «Et surtout, pour la langue littéraire d'abord, mais aussi pour les patois, il importe de confronter les matériaux actuels avec le plus grand nombre possible de documents historiques bien localisés. Sans cet appui solide, les esprits les plus pénétrants s'exposent eux-mêmes à des déconvenues.» (1944: 48)

\section{Conclusion}

À ma connaissance, il n'y a que deux articles, celui de Gilliéron et Mongin (1907) et celui de Brun-Trigaud, Le Berre et Le Dû (2005), qui ont traité de la distribution géolinguistique du mot sel dans l'ensemble de la France. 
Le dernier a corrigé une erreur cartographique chez le premier concernant la forme languedocienne sal. Le présent exposé a pu montrer la pertinence de quelques principes géolinguistiques tels que la distribution des aires latérales, mais aussi celle de la continuité temporelle et géographique des dialectes et de l'apport des documents métalinguistiques.

Notre argumentation s'est concentrée sur la problématique de la consonne /-1/ finale. D'après notre hypothèse, la chute de /-1/ finale est attestée dès la fin du XIII e siècle sous la plume d'un auteur parisien. Il faut ne pas oublier que le maintien de la consonne finale était toujours la norme dans les poèmes écrits par les lettrés, la suppression de /-1/ étant propre au phonétisme vulgaire. Cette tendance a persisté pendant plusieurs siècles, et il ressort du témoignage des grammairiens que la chute de /-1/ ne traduit pas une variation aléatoire, mais sociolinguistique. La variation est rapprochée par les grammairiens des différences entre couches sociales : - el pour la couche haute et -é pour la couche basse. À notre avis, elle doit cependant être regardée comme une variation entre registres : il est probable qu'un même locuteur se servait des deux variantes suivant la situation de communication. L'absence de /-1/ coexistait donc avec son maintien. Depuis le $\mathrm{XIX}^{\mathrm{e}}$ siècle, sous la pression croissante de la capitale et du prestige de l'orthographe, sel va conquérir en français standard et aussi dans les dialectes de la France du Nord. Dans l'ALB, la forme sé ne pourra survivre que dans des dialectes parlés en Puisaye et dans le Morvan, qui semblent rester à l'abri des vagues de standardisation du français (Kawaguchi, 2001 : 257; Kawaguchi, 2017 : 241-242).

\section{RÉFÉRENCES BIBLIOGRAPHIQUES}

BeC Pierre, 1983, Manuel pratique d'occitan moderne ( $2^{\circ}$ éd.), Paris, Picard.

Berschin Helmut, Felixberger Josef \& Goebl Hans, 2008, Französische Sprachgeschichte: 2., überarbeitete Auflage, Hildesheim, Georg Olms Verlag.

Brun-Trigaud Guylaine, Le Berre Yves \& Le Dô Jean, 2005, Lectures de l'Atlas linguistique de la France de Gilliéron et Edmont. Du temps dans l'espace, Paris, Éditions du CTHS.

BRUn-Trigaud Guylaine, 2016, «Vers un renouveau des atlas linguistiques régionaux?», Géolinguistique, n 16, p. 7-19.

DAlberA Jean-Philippe, 2007, «Linguistic Atlases: Objectives, Methods, Results, Prospects», dans Y. Kawaguchi et al. (éds), Corpus-Based Perspectives in Linguistics, Amsterdam/Philadelphie, John Benjamins, p. 39-54.

Dauzat Albert, 1944, Géographie linguistique - 9 cartes, Paris, Flammarion.

De la Chaussée François, 1989, Initiation à la phonétique historique de l'ancien français, Paris, Klincksieck. 
ERnst Gerhard, Gesprochenes Französisch zun Beginn des 17. Jahrhunderts. Direkte Rede in Jean Héroards «Histoire particulière de Louis XIII» (1605-1610), Tübingen, Max Niemeyer Verlag.

Faral Edmond \& Bastin Julia, 1985 [1959], Euvres complètes de Rutebeuf, t. 1, Paris, Éditions A. et J. Picard.

Fouché Pierre, 1961, Phonétique historique du français, vol. III : Les consonnes et index général, Paris, Klincksieck.

Gilliéron Jules \& Mongin Jules, 1906, «Études de géographie linguistique, I. Déchéances sémantiques : Oblitare», Revue de philologie française et de littérature, $\mathrm{n}^{\circ} 20$, p. 81-110.

Gilliéron Jules \& Mongin Jules, 1907, «Études de géographie linguistique, IX. Le sel; les aires disparues », Revue de philologie française et de littérature, $\mathrm{n}^{\circ} 21$, p. 292-296.

Guéssard François \& Meyer Paul, 1861, Aye d'Avignon, Chanson de geste, Paris, F. Vieweg.

KawAGUChI Yuji, 2001, «À propos de la francisation des dialectes bourguignons », dans J. Foyard et P. Monnere (éds), Mélanges de dialectologie, toponymie, onomastique offerts à G. Taverdet, Dijon, vol. 1, p. 255-274.

Kawaguchi Yuji, 2017, «How Can We Depict Standardization in the Linguistic Atlas? Case Study of Champagne and Brie (ALCB)», Philologica Jassyensia, vol. 24, no 2, p. 237-250.

Lauwers Peter, Simoni-Aurembou Marie-Rose \& Swiggers Pierre, 2002, Géographie linguistique et biologie du langage : autour de Jules Gilliéron, Louvain, Paris, Peeters, coll. «Orbis Supplementa», n 20.

Meyer-LüBKe Wilhelm, 1895, Grammaire des langues romanes, traduction française par A. Doutrepont \& G. Doutrepont, Paris, Éd. H. Welter.

Pope Mildred K., 1934, From Latin to Modern French, with Especial Consideration of Anglo-Norman; Phonology and Morphology, Manchester University Press.

PotTier Bernard, 1968, «La situation en France», dans A. Martinet (éd.), Le Langage, Paris, La Pléiade, p. 1144-1161.

Regula Moritz, 1955, Historische Grammatik des Französischen. Bd. I, Lautlehre, Heidelberg, Carl Winter Universitätsverlag.

Rosenqvist Arvid, 1919, «Limites administratives et division dialectale de la France», Neuphilologische Mitteilungen, $\mathrm{n}^{\circ}$ 20, p. 87-119.

Scheler Auguste, 1868, Dits de Watriquet de Couvin, Bruxelles, Comptoir universel d'imprimerie et de librairie.

Simoni-Aurembou Marie-Rose, 2004, «Les Atlas linguistiques de la France par régions (1939-1970)», Flambeau, n 30, Tokyo University of Foreign Studies, p. 1-22.

STRAKA Georges, 1952, «La prononciation parisienne ses divers aspects (Introduction à l'étude de la prononciation du français moderne)», Bulletin de la Faculté de Lettres de l'Université de Strasbourg, n 30, p. 212-225 et 239-253.

STRAKA Georges, 1981, «Sur la formation de la prononciation française d'aujourd'hui », Travaux de littérature et de linguistique, vol. 19, nº 1, p. 161-248. 
TAVERDET Gérard, 1973, «Patois et français régional en Bourgogne», Ethnologie française, vol. 3, no 3-4, p. 317-328.

TAVERDET Gérard, 1975-1980, Atlas linguistique et ethnographique de Bourgogne $(A L B)$, vol. I-III, Paris, Éditions du CNRS.

ThibAult André, 2017, «Le sort des consonnes finales en français, en galloroman et en créole : le cas de moins », Revue de linguistique romane, $\mathrm{n}^{\circ}$ 81, p. 5-41.

ZINK Michel (éd.) 1989, Rutebeuf Euvres complètes, tome I, Paris, Classiques Garnier.

\section{Témoignages métalinguistiques}

BOyer Abel, 1794, The Complete French Master for Ladies and Gentlemen (new edition), Londres, T. Longman et al.

Buffier Claude, 1709, Grammaire françoise, Paris, Chez Nicolas le Clerc et al.

Buffier Claude, 1732, Cours de sciences sur des principes nouveaux et simples, Paris, Guillaume Cavelier \& Pierre-François Giffart.

Chifflet Laurent, 1680, Essay d'une parfaite grammaire de la langue françoise ( $6^{\mathrm{e}}$ et dernière édition), Bruxelles.

Estienne Henri, 1582, Hypomneses de Gall. lingua.peregrinis eam discentibus necessariae quaedam vero ipsis etiam Gallis multum profuturae, Stephanus.

FÉRAud Jean-François, 1761, Dictionnaire grammatical de la langue françoise, Avignon, la veuve Girard.

FéLINE Adrien, 1851, Dictionnaire de la prononciation de la langue française, Paris, Firmin Didot Frères.

IRSON Claude, 1662, Nouvelle méthode pour apprendre facilement les principes et la pureté de la langue françoise, Paris, Chez P. Baudouin.

LA Touche Pierre DE, 1760, L'art de bien parler françois ( $7^{\mathrm{e}}$ éd.), Amsterdam/ Leipzig, Chez Arkstee \& Merkus.

Malvin-Cazal Joseph DE, 1846, Prononciation de la langue françoise au XIX siècle, Paris, Imprimerie nationale.

MAuvillon Eléazar DE, 1754, Cours complet de la langue françoise, Dresde.

Michaelis Hermann \& Passy Paul, 1914, Dictionnaire phonétique de la langue française [1897], Berlin, Carl Meyer.

Oudin Antoine, 1640, Grammaire françoise, rapportée au langage du temps $\left(2^{\mathrm{e}}\right.$ éd.), Paris, Chez Antoine de Sommaville.

Palsgrave John, 1852 [1530], L'éclaircissement de la langue française, publié pour la première fois en France par F. Génin, Paris, Imprimerie nationale.

RÉGNIER-DESMARAIS François-Séraphin, 1706, Traité de la grammaire françoise, Bruxelles, Eugène Henry Fricx.

Saint-Liens Claude DE, 1566, De Pronuntiatione linguae gallicae, Londres, Thomas Vautrollerius Typographus.

Thurot Charles, 1881, De la prononciation française depuis le commencement $d u$ XVI ${ }^{e}$ siècle d'après les témoignages des grammairiens, 2 tomes, Paris, Imprimerie nationale. 


\section{Ressources en ligne}

Französisches etymologisches Wörterbuch (FEW). Disponible sur <https://apps. atilf.fr/lecteurFEW/index.php/page/view $>$.

Trésor de la langue française informatisé (TLFi). Disponible sur $<\mathrm{http}$ ://atilf.atilf. fr/tlf.htm >. 